L. E. Snyder ${ }^{1}$, J. M. Hollis ${ }^{2}$, L. M. Ziurys ${ }^{3}$, and Y. -J. Kuan ${ }^{1}$

${ }^{1}$ Astronomy Dept., Univ. of Illinois; ${ }^{2}$ Space Data \& Computing Div., NASA/GSFC; ${ }^{3}$ Chemistry Dept., Arizona State Univ.

ABSTRACT. Interferometric observations of the HNO $3.68 \mathrm{~mm}$ line in Sgr B2 show clumping and some distribution similarities with HNCO. A search for the HNO $1.23 \mathrm{~mm}$ line with the NRAO 12 -m telescope was unsuccessful.

\title{
1. Introduction and Observations
}

HNO studies are important because some compounds with the $\mathrm{N}-\mathrm{O}$ bond appear to be underabundant compared to ion-molecule predictions, and the exact location of $\mathrm{N}$ compounds in cloud clumps is not well known. The identification of interstellar HNO was confirmed recently by FCRAO and BIMA array observations (Hollis et al. 1991; Snyder et al. 1991). New BIMA array maps of the HNO $1_{01}-0_{00}$ line at $81,477.56 \mathrm{MHz}$ (3.68 mm) around Sgr B2 are presented here (Figs. 1 and 2); these new maps used 20 baselines ( 5 configurations in B-array and 3 in C-array).

In addition, the NRAO $12-\mathrm{m}$ telescope equipped with a DSB SIS receiver was used to search $\mathrm{Sgr} B 2(\mathrm{M})$ for the HNO $3_{03}-2_{02}$ line at $244,364.09 \mathrm{MHz}$ $(1.23 \mathrm{~mm})$. These observations were conducted in March and April, 1991.

\section{Results and Conclusions}

At $3.68 \mathrm{~mm}, 5$ HNO emission clumps and 1 absorption source ( $\mathrm{Sgr}$ B2(M)) were detected with the BIMA array. We note that HNO observations with a 6-m BIMA single antenna (2' HPBW) show that the emission peaks at 69 $\mathrm{km} / \mathrm{s}$ in $\mathrm{Sgr} \mathrm{B} 2(\mathrm{M})$, but $30^{\prime \prime}$ south in Sgr B2(OH) it peaks at $64 \mathrm{~km} / \mathrm{s}$. This suggests that a typical single-dish Sgr B2(M) HNO spectrum (not shown) is dominated by clumps with higher velocity than those near $\mathrm{Sgr} \mathrm{B} 2(\mathrm{OH})$. The main HNO features generally agree with BIMA maps of the HNCO $4_{04}-3_{03}$ line at $87,925.24 \mathrm{MHz}$ near $68 \mathrm{~km} / \mathrm{s}$, but the HNCO is much stronger.

The HNO $3_{03}-2_{02}$ line was not detected in $\mathrm{Sgr}$ B2(M), with an upper limit of $\mathrm{T}_{\mathrm{A}}{ }^{*}<0.05 \mathrm{~K}(3 \sigma)$. Because this limit is comparable to the HNO intensities at lower frequencies, it suggests weak excitation.

Support came from NASA PO S-46462-E, and the Laboratory for Astronomical Imaging with funds from the University of Illinois and NSF AST 90-24603. 
SGRB2 HNO MAPS

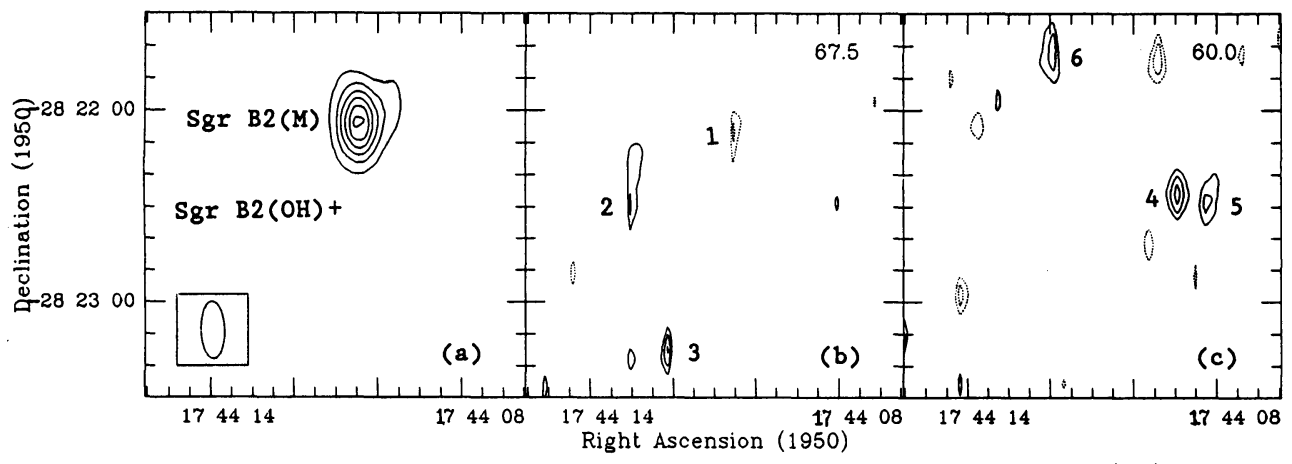

Figure 1. (a) $\mathrm{Sgr} \mathrm{B} 2(\mathrm{M}) 3.68 \mathrm{~mm}$ continuum. Contour intervals: $1.0 \mathrm{Jy} / \mathrm{bm}$. Peak $=6.11 \mathrm{Jy} / \mathrm{bm}$. The inset shows the $17 " .95 \times 7 " .41$ beam. (b) HNO at $67.5 \mathrm{~km} / \mathrm{s}$ (averaged over $10 \mathrm{~km} / \mathrm{s}$ intervals). Contour intervals: -0.25 , -0.30 (dots); and $0.25,0.30,0.35 \mathrm{Jy} / \mathrm{bm}$ (solid). Peak $=0.36 \mathrm{Jy} / \mathrm{bm}$. (c) HNO at $60.0 \mathrm{~km} / \mathrm{s}$ (averaged over $7 \mathrm{~km} / \mathrm{s}$ intervals). Contour intervals: $-0.3,-0.4$ (dots); and $0.3,0.4,0.5 \mathrm{Jy} / \mathrm{bm}$ (solid). Peak $=0.56 \mathrm{Jy} / \mathrm{bm}$.
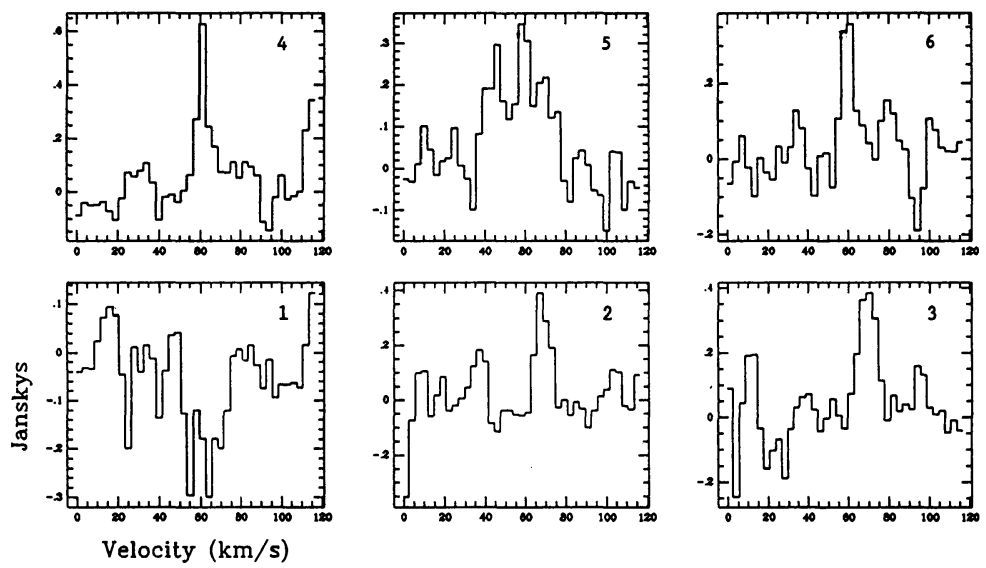

Figure 2. Spectra of the HNO $3.68 \mathrm{~mm}$ line constructed from data cubes corresponding to numbered clumps in Figure 1. Abscissa: LSR radial velocity $(\mathrm{km} / \mathrm{s})$. Ordinate: intensity (Jy/beam).

\section{References}

Hollis, J. M., Snyder, L. E., Ziurys, L. M., and McGonagle, D.

(1991) 'Interstellar HNO: confirming the identification', in A. D. Haschick and P. T. P. Ho (eds), Skylines, ASP Conference Series 16, San Francisco, pp. 407-12.

Snyder, L.E., Kuan, Y.-J., and Pratap, P. (1991) 'Millimeter wavelength molecular maps of the clumped gas around Sgr B2(OH)', Ibid., pp. 191-6. 